\title{
Integral Attacks on Feistel-SP Structure Block Cipher
}

\author{
Yanjun Li \\ The Department of Information Security \\ Beijing Electronic Science and Technology Institute \\ Beijing, P.R. China \\ liyjwuyh@163.com
}

\begin{abstract}
In this paper, a method is presented to extend the length of integral distinguisher of Feistel-SP structure, based on which a new 8-round distinguisher for the block cipher Camellia is proposed. Moreover, integral attacks on round-reduced Camellia without $F L / F L^{-1}$ are improved. We attack 11-round Camellia-128 with the data complexity of $2^{120}$ and the time complexity of $2^{125.5}$, and attack 12 -round Camellia-256 with the data complexity of $2^{120}$ and the time complexity of $2^{214.3}$. These attacks are the best integral attacks on round-reduced Camellia so far.
\end{abstract}

Keywords-block cipher, distinguisher, integral attack, camellia, partial sum technique

\section{INTRODUCTION}

The block cipher Camellia was proposed by NTT and Mitsubishi in $2000^{[1]}$. It is based on Feistel structure with SP-type $\mathrm{F}$ function and $F L / F L^{-1}$ functions layers, and it supports the block length of 128 bits and a variable key length of $128 / 192 / 256$ bits. Camellia was accepted by ISO/IEC as an international standard ${ }^{[6]}$. It is also a winner of NESSIE, CRYPTREC project and IETF. The security of Camellia was initially analyzed by the algorithm designers. Efficient attacks on Camellia include linear cryptanalysis $^{[14]}$, differential cryptanalysis ${ }^{[13,14]}$, impossible differential cryptanalysis ${ }^{[15,}{ }^{17]}$, truncated differential cryptanalysis ${ }^{[7,9,15]}$, higher order differential cryptanalysis $^{[5]}$, collision attack ${ }^{[16]}$ and Square attack ${ }^{[3,19}$, ${ }^{20]}$. The best attacks on Camellia without $F L / F L^{-1}$ function layer were impossible differential cryptanalysis ${ }^{[18]}$, which can attack 12-round Camellia-128 and 16- round Camellia-256 without $F L / F L^{-1}$.

Integral attack was extended from Square attack, which is one of the best attacks on AES ${ }^{[2]}$. Ferguson et al. in [4] improved this attack to 8 rounds version of Rijndael-128 with the partial sum technique and the herd technique. Knudsen and Wagner first proposed the definition of integral and analyzed it as a dual to differential attacks particularly applicable to block ciphers with bijective components ${ }^{[8]}$. Several years later, Reza Z'aba et al. presented bit pattern based integral attack ${ }^{[12]}$. The integral attack applied to many kinds of block ciphers so far, such as Rijndael ${ }^{[11]}$, ARIA ${ }^{[10]}$, and Serpent ${ }^{[12]}$. Higher order differential attack and Square attack are different from integral attack. However, the length of their distinguisher can be extended by using the integral property. In this paper a method is presented to extend the length of Camellia's distinguisher, based on which the effect of integral attack will be improved. Moreover, this method can also be used even on any Feistel-SP structure. Then a new 8-round distinguisher of Camellia without $F L / F L^{-1}$ is proposed. Finally, we attack

\author{
Wenling Wu, Lei Zhang, and Liting Zhang \\ State Key Laboratory of Information Security \\ Institute of Software, Chinese Academy of Sciences \\ Beijing, P.R. China
}

11-round Camellia-128 with the data complexity of $2^{120}$ and the time complexity of $2^{125.5}$, and 12-round Camellia-256 with the data complexity of $2^{120}$ and the time complexity of $2^{214.3}$. The result is the best integral attack on round-reduced Camellia so far.

This paper is organized as follows: Section II provides a brief description of preliminaries. Section III describes a method to extend the length of the distinguisher. Section IV describes the attacks on 11/12-round Camellia. Finally, Section V concludes this paper.

\section{PRELIMINARIES}

\section{A. Description of Camellia}

Camellia is a Feistel-SP style block cipher with $F L / F L^{-1}$ layers, and the number of rounds are $18 / 24 / 24$ corresponding to key length of 128/192/256 bits. Additionally, $F L / F L^{-1}$ function is inserted every 6 rounds (Fig.1). The round keys are derived from the master key by means of key scheduling. The key schedule constants are listed in Table 1. In this paper the input and output of round function are treated as two 8-byte vectors over $F_{2^{8}}^{8}$.

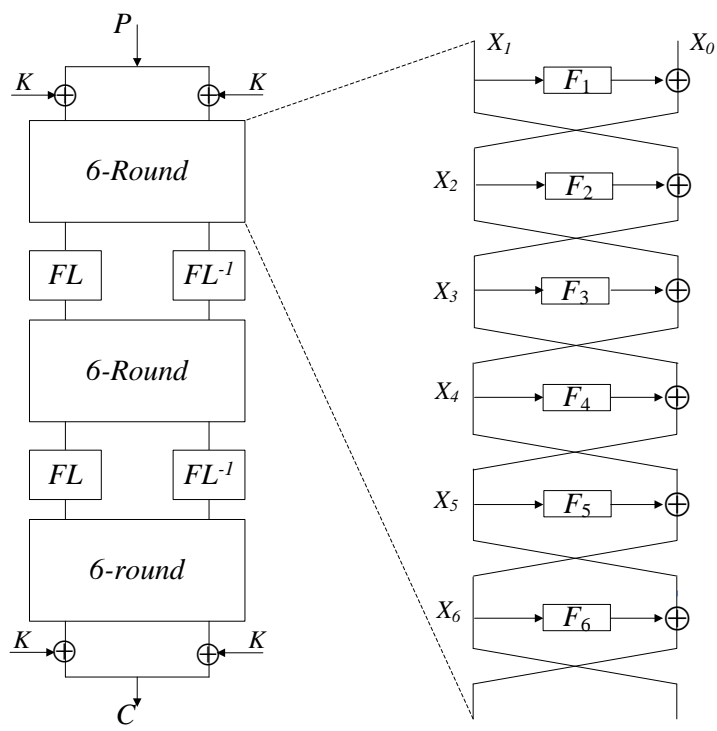

Fig. 1. The Structure of Camellia-128

The round function of Camellia includes three basic operations: Round Key Addition, Substitution Layer and Diffusion Layer (Fig.2). These three basic operations are defined as follows:

Round Key Addition (RKA): The 64-bit round key is Xored to the state.

Substitution Layer (SL): A non-linear byte substitution 
operation is applied to each byte of the state independently. In Camellia this is implemented by 4 S-boxes with the relationship as follows.

$$
\begin{aligned}
& s_{2}(a)=s_{1}(a)<<<1 ; \\
& s_{3}(a)=s_{1}(a)>>>1 ; \\
& s_{4}(a)=s_{1}(a<<<1) .
\end{aligned}
$$

Diffusion Layer (DL): The diffusion layer is a function $P: F_{2^{8}}^{8} \rightarrow F_{2^{8}}^{8}$, which is given by

$$
\begin{aligned}
& m_{1}=x_{1} \oplus x_{3} \oplus x_{4} \oplus x_{6} \oplus x_{7} \oplus x_{8} \\
& m_{2}=x_{1} \oplus x_{2} \oplus x_{4} \oplus x_{5} \oplus x_{7} \oplus x_{8} \\
& m_{3}=x_{1} \oplus x_{2} \oplus x_{3} \oplus x_{5} \oplus x_{6} \oplus x_{8} \\
& m_{4}=x_{2} \oplus x_{3} \oplus x_{4} \oplus x_{5} \oplus x_{6} \oplus x_{7} \\
& m_{5}=x_{1} \oplus x_{2} \oplus x_{6} \oplus x_{7} \oplus x_{8} \\
& m_{6}=x_{2} \oplus x_{3} \oplus x_{5} \oplus x_{7} \oplus x_{8} \\
& m_{7}=x_{3} \oplus x_{4} \oplus x_{5} \oplus x_{6} \oplus x_{8} \\
& m_{8}=x_{1} \oplus x_{4} \oplus x_{5} \oplus x_{6} \oplus x_{7}
\end{aligned}
$$

\section{B. Notations}

In the following, we introduce some notations used in this paper. The plaintext are denoted as $\left(X_{1}, X_{0}\right)$, where $X_{i}=\left(x_{i, 1}, x_{i, 2}, \cdots, x_{i, 8}\right), \quad i=0, \ldots, r-1$. Other notations that will be used in this paper are described as follows:

$B_{r}$ : the output of RKA in $r$-th round.

$O_{r}:$ the output of SL in $r$-th round.

$M_{r}:$ the output of DL in $r$-th round.

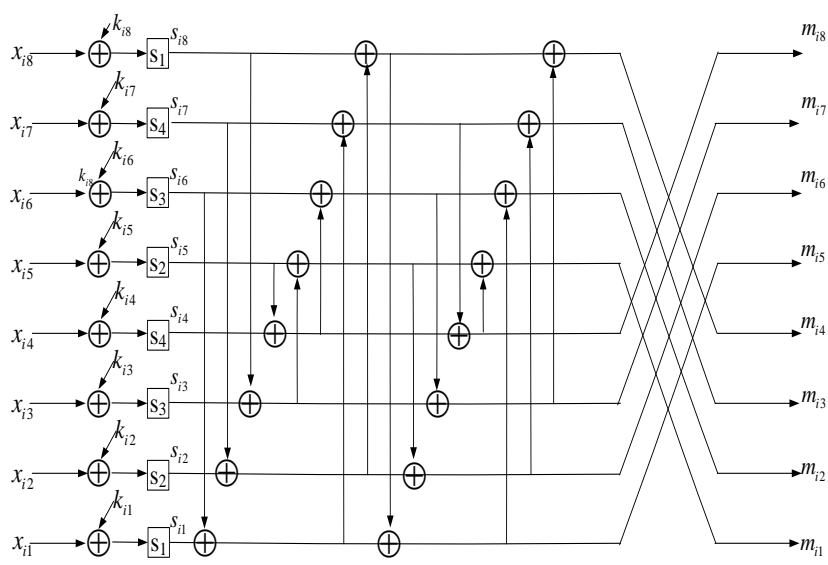

Fig. 2. The Round Function of Camellia

$K_{r}$ : the subkey of the $r$-th round.

$b_{r, i}:$ the $i+1-t h$ byte of $B_{r}$.

$o_{r, i}:$ the $i+1-t h$ byte of $O_{r}$.

$m_{r, i}:$ the $i+1-t h$ byte of $M_{r}$.

$k_{r, i}:$ the $i+1-t h$ byte of $K_{r}$.

$\lambda_{i}:$ the active bytes.

C. Higher Order Integral Attack and the Partial Sum Technique

Higher Order Integral Attack. The integral attack has many interesting features. It can saturate S-Box Layer and Round Key Addition Layer will not affect this property of saturation. However, the linear transformation influences the length of the integral distinguisher. Integral attack considers a particular collection of $m$ bytes in the plaintexts and ciphertexts. In [8], Knudsen and Wagner also generalized this approach to higher order integrals: the original set to consider becomes a set of $m^{d}$ vectors which differ in $d$ components where the sum of this set is predictable after a certain number of rounds. The sum of this set is called a $d^{\text {th }}$-order integral. In this paper we not only pay attention to the sum, but also to the appearing times of the sum value.

The Partial Sum Technique. In our attack we will use the partial sum technique. For a value $c_{0}, c_{1}, c_{2}, \ldots, c_{l}$, we define

$$
x_{u}:=\sum_{j=0}^{u} S_{j}\left[c_{j} \oplus k_{j}\right] .
$$

Guessing the values of $k_{0}$ and $k_{1}$,we will complete the transformation

$$
\left(c_{0}, c_{1}, c_{2}, \ldots, c_{l}\right) \rightarrow\left(x_{1}, c_{2}, \ldots, c_{l}\right) .
$$

Guessing the values of $k_{i}$, we will complete the transformation

$$
\left(x_{i-1}, c_{i}, c_{i+1}, \ldots, c_{l}\right) \rightarrow\left(x_{i}, c_{i+1}, \ldots, c_{l}\right) .
$$

In order to obtain the value of $x_{l}, l-1$ steps of processing are required. If $c_{i} \mathrm{~s}$ are in byte pattern, the time complexity of the count of $x_{l}$ is $2^{8 l} \times 2^{16} \times(l-1)$ times S-box lookups. For the details of the complexities of each step, the readers can refer to [4].

\section{INTEGRAL DISTINGUISHERS BASED ON FEISTEL-SP STRUCTURE}

In this section we first explain how to construct a 2nd-order 5-round integral distinguishers (Sec. 2.1), then introduce a method to extend the length of integral distinguishers and the proof is also given in detail (Sec. 2.2).

\section{A. The 2nd-Order 5-Round Integral Distinguisher}

The idea of constructing a 2nd-order 5-round integral distinguisher is like that of constructing 5-round higher order differential distinguishers in [5].

Lemma $1^{[5]}$. Let the bytes of $x_{0,1}, x_{0,2}$ are active, and other bytes of $X_{0}, X_{1}$ are constants, each value of $t$ appears even times.

$$
t=s_{3}\left(x_{6,6} \oplus k_{6,6}\right) \oplus\left[P^{-1}\left(X_{7}\right)\right]_{6} .
$$

\section{B. A Method to Extend the Length of Integral Distinguisher}

In the structure of Feistel-SP, the Xor operation and the permutation $P$ are linear transformations (Fig.3), which can influence the general integral property and also can be used to extend the integral distinguisher. Let some bytes of $X_{0}$ be active, and the bytes of $X_{1}$ be constant, and then the input of a known $t$-round integral distinguisher is $\left(X_{1}, X_{0}\right)$. Now we extend it backward 
by one round using the following formula:

$$
\begin{gathered}
X_{1}=F\left(X_{0}\right) \oplus X_{-1}=P \circ S\left(K\left(X_{0}\right)\right) \oplus X_{-1} . \\
=P\left[S\left(K\left(X_{0}\right)\right) \oplus P^{-1}\left(X_{-1}\right)\right]
\end{gathered}
$$

Choose $X_{-1}$, which satisfying that the bytes of $P^{-1}\left(X_{-1}\right)$ corresponding to the no-constant bytes of $S\left(K\left(X_{0}\right)\right)$ are active and other bytes are constants. Such $\left(X_{0}, X_{-1}\right)$ will lead to several sets of $\left(X_{1}, X_{0}\right)$ after one-round encryption, and we will obtain a $t+1$-round integral distinguisher. The related proof will be presented in Lemma 2.

Take Camellia for example, the input of a $2^{\text {nd }}$ order 5 -round distinguisher is $\left(X_{1}, X_{0}\right)$, where the bytes of $x_{0,1}, x_{0,2}$ are active and other bytes are constants. Extending three more rounds forward and we will obtain the following:

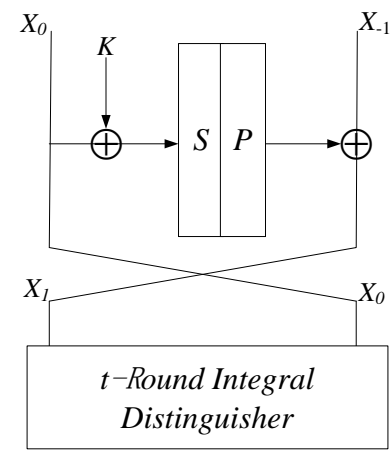

Fig. 3. The Feistel-SP Structure

$$
\begin{aligned}
& X_{-1}=P\left(\lambda_{2}, \lambda_{3}, c, c, c, c, c, c\right), \\
& X_{-2}=P\left(\lambda_{4}, \lambda_{5}, \lambda_{6}, \lambda_{7}, \lambda_{8}, \lambda_{9}, c, \lambda_{10}\right), \\
& X_{-3}=P\left(\lambda_{11}, \lambda_{12}, \lambda_{13}, \lambda_{14}, \lambda_{15}, \lambda_{16}, \lambda_{17}, \lambda_{18}\right) .
\end{aligned}
$$

where $\lambda_{i} s, 0 \leq i \leq 18$ are active bytes. Because all bytes of $X_{-3}$ are active, we can't further improve it any more. Then the following lemma is obtained.

Lemma 2. Let the 7th byte of $P^{-1}\left(X_{-2}\right)$ be constant and other 15 bytes of $\left(P^{-1}\left(X_{-2}\right), X_{3}\right)$ be active. After 3 rounds iterative encryption we will obtain $2^{104}$ sets and in each set the bytes of $x_{0,1}, x_{0,2}$ are active and other bytes are constants.

Proof. We will proof this lemma in three steps as follows:

1) The $2^{32}$ values of $\left(X_{1}, X_{-1}\right)$ will lead to $2^{16}$ sets of $\left(X_{1}, X_{0}\right)$ after one-round encryption, in each set the first two bytes of $X_{1}$ are active and other bytes are constant.

The proof of this step is just like that of example 1 and omitted here.

2) The $2^{72}$ values of $\left(X_{-1}, X_{-2}\right)$ will lead to $2^{40}$ sets of $\left(X_{1}, X_{-1}\right)$ after one round encryption. In each set $x_{1,0}, x_{1,1}$ and the first two bytes of $P^{-1}\left(X_{-1}\right)$ are active, and other bytes are constants.

Let all active bytes of $\left(P^{-1}\left(X_{-1}\right), P^{-1}\left(X_{-2}\right)\right)$ be denoted as $x_{1}, x_{2}, x_{3}, x_{4}, x_{5}, x_{6}, x_{7}, x_{8}, x_{9}$, and all constants be denoted as 0 . Let the active bytes of $\left(X_{1}, P^{-1}\left(X_{-1}\right)\right)$ be denoted as $y_{3}, y_{4}, y_{1}, y_{2}$. One round encryption will be decrypted as the following equations.

$$
\left\{\begin{array}{l}
x_{1}=y_{1} \\
x_{2}=y_{2} \\
s_{1}\left(x_{1} \oplus k_{1}\right) \oplus x_{3}=y_{4} \\
s_{2}\left(x_{1} \oplus x_{2} \oplus k_{2}\right) \oplus x_{4}=y_{3} \\
s_{3}\left(x_{1} \oplus x_{2} \oplus k_{3}\right) \oplus x_{5}=y_{3} \oplus y_{4} \\
s_{4}\left(x_{2} \oplus k_{4}\right) \oplus x_{6}=y_{3} \oplus y_{4} \\
s_{2}\left(x_{1} \oplus x_{2} \oplus k_{5}\right) \oplus x_{7}=y_{3} \oplus y_{4} \\
s_{3}\left(x_{2} \oplus k_{6}\right) \oplus x_{8}=y_{4} \\
s_{1}\left(x_{1} \oplus k_{8}\right) \oplus x_{9}=y_{3}
\end{array}\right.
$$

Simplifying the above equations, we will obtain the equivalent equations as follows.

$$
\begin{aligned}
& x_{1}=y_{1} \\
& x_{2}=y_{2} \\
& s_{1}\left(x_{1} \oplus k_{1}\right) \oplus x_{3}=y_{4} \\
& s_{2}\left(x_{1} \oplus x_{2} \oplus k_{2}\right) \oplus x_{4}=y_{3} \\
& s_{1}\left(x_{1} \oplus k_{1}\right) \oplus x_{3} \oplus s_{2}\left(x_{1} \oplus x_{2} \oplus k_{2}\right) \oplus x_{4} \\
& \oplus s_{3}\left(x_{1} \oplus x_{2} \oplus k_{3}\right) \oplus x_{5}=0 \\
& s_{1}\left(x_{1} \oplus k_{1}\right) \oplus x_{3} \oplus s_{2}\left(x_{1} \oplus x_{2} \oplus k_{2}\right) \oplus x_{4} \\
& \oplus s_{4}\left(x_{2} \oplus k_{4}\right) \oplus x_{6}=0 \\
& s_{1}\left(x_{1} \oplus k_{1}\right) \oplus x_{3} \oplus s_{2}\left(x_{1} \oplus x_{2} \oplus k_{2}\right) \oplus x_{4} \\
& \oplus s_{2}\left(x_{1} \oplus x_{2} \oplus k_{5}\right) \oplus x_{7}=0 \\
& s_{1}\left(x_{1} \oplus k_{1}\right) \oplus x_{3} \oplus s_{3}\left(x_{2} \oplus k_{6}\right) \oplus x_{8}=0 \\
& s_{2}\left(x_{1} \oplus x_{2} \oplus k_{2}\right) \oplus x_{4} \oplus s_{1}\left(x_{1} \oplus k_{8}\right) \oplus x_{9}=0
\end{aligned}
$$

According to the simplified equations above, we find that there is only one solution. For $2^{32}$ values of $y_{3}, y_{4}, y_{1}, y_{2}$, we get a set of $2^{32}$ solutions, and each value of $x_{5}, x_{6}, x_{7}, x_{8}, x_{9}$ is determined by $x_{1}, x_{2}, x_{3}, x_{4}$. After taking over all $2^{40}$ values of $x_{5}, x_{6}, x_{7}, x_{8}, x_{9}, 2^{40}$ sets will be obtained, i.e. $2^{72}$ values of $x_{1}, x_{2}, x_{3}, x_{4}, x_{5}, x_{6}, x_{7}, x_{8}, x_{9}$ leads to $2^{40}$ sets after one round encryption, in each set the bytes of $y_{1}, y_{2}, y_{3}, y_{4}$ are active.

3) The $2^{120}$ values of $\left(X_{-2}, X_{-3}\right)$ will lead to $2^{48}$ sets of $\left(X_{-1}, X_{-2}\right)$ after one round encryption, and in each set the nine bytes of $\left(P^{-1}\left(X_{-2}\right), P^{-1}\left(X_{-1}\right)\right)$ are active, and other bytes are constants.

The proof of this step is just like that of the above step.

After processing the three steps above, we will obtain $2^{104}$ sets of $\left(X_{1}, X_{0}\right)$ from $2^{120}$ values of $\left(X_{-2}, X_{-3}\right)$ described in the lemma 2 . In each set the bytes of $x_{0,1}, x_{0,2}$ are active and other bytes are constant. 
Utilizing Lemma 1 and Lemma 2, we can construct a new $15^{\text {th }}$ order 8 -round distinguisher of Camellia as depicted in Theorem 1 .

Theorem 1. Let $\left(X_{1}, X_{0}\right)$ be the input of Camellia without $F L / F L^{-1}$. If the bytes of $P^{-1}\left(X_{1}\right)_{7}$ are constants and other bytes of $\left(X_{1}, X_{0}\right)$ take all values of $F_{2^{8}}^{15}$, then each value of $t=s_{3}\left(x_{9,6} \oplus \quad k_{9,6}\right) \oplus P^{-1}\left(X_{10}\right)_{6} \quad$ will appear even times.

\section{ATTACKS ON ROUND-REDUCED CAMELLIA}

\section{A. Integral Attack on 10/11-Round Camellia-128}

Based on the $15^{\text {th }}$-order 8-round distinguisher described above, we will attack 10 rounds of Camellia-128 without $F L / F L^{-1}$ functions now, which is illustrated in Fig.4.

1) Choose a structure of plaintexts $\left(X_{1}, X_{0}\right)$ which satisfies

$$
\begin{aligned}
& X_{1}=P\left(\lambda_{4}, \lambda_{5}, \lambda_{6}, \lambda_{7}, \lambda_{8}, \lambda_{9}, c, \lambda_{10}\right), \\
& X_{0}=P\left(\lambda_{11}, \lambda_{12}, \lambda_{13}, \lambda_{14}, \lambda_{15}, \lambda_{16}, \lambda_{17}, \lambda_{18}\right),
\end{aligned}
$$

where $\lambda_{i}(4 \leq i \leq 18)$ are active bytes, and $c$ is a constant. $\left(X_{1}, X_{0}\right)$ takes all values of $F_{2^{8}}^{15}$. Encrypt all these plaintexts and set $2^{56}$ counters for the seven bytes of $x_{11,6}, x_{10,2}, x_{10,3}, x_{10,5}, x_{10,6}, x_{10,7}, x_{10,8}$. Once a 56-bit value is obtained, the corresponding counter is increased by one.

2) For the $2^{56}$ values of ciphertexts, there are at most $2^{56}$ values in bytes of $x_{11,6}, x_{10,2}, x_{10,3}, x_{10,5}, x_{10,6}$, $x_{10,7}, x_{10,8}$. We choose those values that counters are odd times (the Xor value of the same value is zero).

Guessing the key bytes of $k_{10,2}, k_{10,3}, k_{10,5}, k_{10,7}, k_{10,8}$ and $k_{9,6}$, we do a partial decrypt to the single value of $t$.In this phase we need the partial sum technique in order to reduce the work factor of computing the value of $s_{3}\left(x_{9,6} \oplus k_{9,6}\right)$.

$$
\begin{aligned}
t & =\left[P^{-1}\left(X_{10}\right)\right]_{6} \oplus s_{3}\left(x_{9,6} \oplus k_{9,6}\right) \\
& =\left[P^{-1}\left(x_{10,2}, x_{10,3}, x_{10,5}, x_{10,6}, x_{10,8}\right)\right]_{6} \oplus s_{3}\left[s_{2}\left(x_{10,2} \oplus k_{10,2}\right)\right. \\
& \oplus s_{3}\left(x_{10,3} \oplus k_{10,3}\right) \oplus s_{2}\left(x_{10,5} \oplus k_{10,5}\right) \oplus s_{4}\left(x_{10,7} \oplus k_{10,7}\right) \\
& \left.\oplus s_{1}\left(x_{10,8} \oplus k_{10,8}\right) \oplus x_{11,6} \oplus k_{9,6}\right] .
\end{aligned}
$$

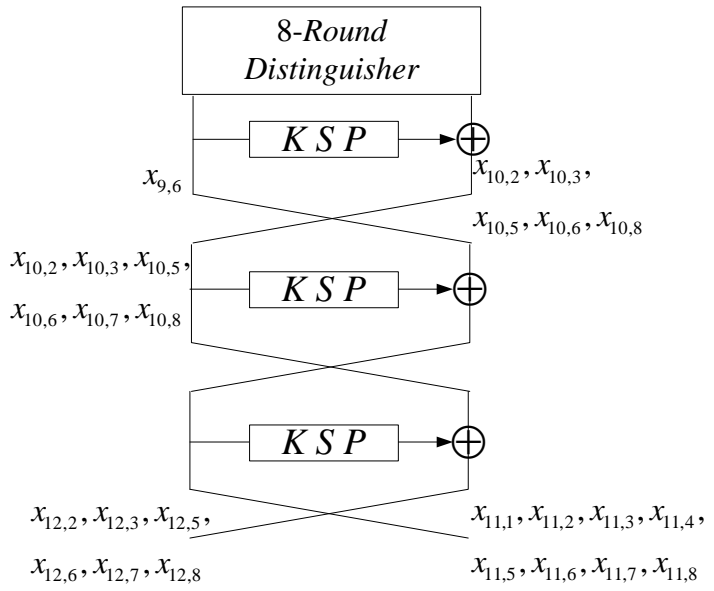

Fig.4. Integral Attacks on Round-reduced Camellia

For the values of $x_{11,6}, x_{10,2}, x_{10,3}, x_{10,5}, x_{10,7}, x_{10,8}$, the number of which appear odd times, we do the following steps:

(a) Guess the two bytes of $k_{10,2}$ and $k_{10,3}$, and we obtain the corresponding 5-byte value $\left(x_{1}, x_{10,5}, x_{10,7}, x_{10,8}, x_{11,6}\right)$.

(b)-(e) Guess the value of $k_{10,5}, k_{10,7}, k_{10,8}, k_{9,6}$ respectively, and then we obtain 1 byte value $x_{5}$.

3) The values of $x_{5}$ and $\left[P^{-1}\left(X_{10}\right)\right]_{6}$ are summed over all the encryptions, and check if the value of $t$ appears even times. If each value of $t$ appears even times, the guessed key bytes are correct, otherwise they are wrong.

In Step 1, we choose $2^{120}$ plaintexts and need encrypt $2^{120}$ times. In Step 2-(a), we guess 16 bits key, and process $2^{48}$ ciphertexts, which cost $2^{64}$ S-box applications. Step 2-(b) costs $2^{16} \times 2^{8} \quad \times 2^{40}=2^{64}$ computations at most. This is same as the other phases of Step 2, so the work factor of Step 2 is $2^{64} \times 5$ S-box lookups. There are also additional cost to compute $\left[P^{-1}\left(X_{10}\right)\right]_{6}$ in each phase of Step 2. However, using rough equivalence of $8 \mathrm{~S}$-box applications to one-round encryption with a new key, the complexity to compute $\left[P^{-1}\left(X_{10}\right)\right]_{6}$ can be ignored. For a wrong key, the probability that each value of $t$ appears even times is less than $2^{-169}$. After analyzing a structure of plaintexts, we expect $2^{48} \times 2^{-169}=2^{-121}$ wrong key that would pass Step 2 . So the data complexity of $2^{120}$ is enough and the time complexity

$2^{48} \times 2^{16} \times 5 /\left(2^{3} \times 10\right)=2^{60}$ encryptions.

We can also improve this attack by adding one more round. For 11-round Camellia-128, the key schedule could be used. The total time complexity is $2^{132} /\left(2^{3} \times 11\right) \approx 2^{125.5}$ encryptions.

\section{B. Integral Attacks on Camellia-192/256}

In this subsection, we describe improved integral attacks on 11-round Camellia-192 and 12-round Camellia-256. The key schedule can't be used here. We add 1 round after 10-round Camellia, and guess all bytes of $K_{11}$, and decrypt the last round, where the work factor 
is the equivalent of $2^{64} \times 2^{64}=2^{128} \mathrm{~F}$ function operations and $2^{64} \times 2^{48} \times 2^{48}=2^{160}$ Xor operations. The rest work factor can be ignored, so the main time complexity of the attack on 11 rounds Camellia-192/256 is $2^{160} /\left(2^{6} \times 11\right) \approx 2^{150.5}$. In a similar way the time complexity of the attack on 12 rounds Camellia-256 is $2^{214.3}$. No relation of subkeys can be used.

\section{CONCLUSION}

The improved integral attacks on round-reduced Camellia are described in this paper. We propose a method of forward-extending the length of integral distinguisher, by which a new 8-round integral distinguisher for Camellia is proposed. Then we attack 11-round Camellia-128 and 11/12-round Camellia-192/256 with the partial sum technique. Table 1 summarizes our integral attacks together with the previously known integral-like attacks on Camellia.

Table 1. Results of Integral-like Attacks on Camellia

\begin{tabular}{|l|l|l|l|l|l|}
\hline Camellia- $b$ & Rounds & Method & Data & Time & Notes \\
\hline Camellia-128 & 8 & SA & $2^{48}$ & $2^{116}$ & {$[19]$} \\
\cline { 2 - 6 } & 9 & SLA & $2^{66}$ & $2^{84.8}$ & {$[3]$} \\
\cline { 2 - 6 } & 10 & IntA & $2^{120}$ & $2^{120}$ & Sec3.1 \\
\cline { 2 - 6 } & 11 & IntA & $2^{120}$ & $2^{123.9}$ & Sec3.1 \\
\hline Camellia-192 & 9 & HODC & $2^{21}$ & $2^{188}$ & {$[5]$} \\
\cline { 2 - 6 } & 9 & SA & $2^{60.5}$ & $2^{202}$ & {$[19]$} \\
\cline { 2 - 6 } & 10 & SLA & $2^{66}$ & $2^{167.3}$ & {$[3]$} \\
\cline { 2 - 6 } & 11 & SLA & $2^{66}$ & $2^{211.6}$ & {$[3]$} \\
\cline { 2 - 6 } & 11 & HODC & $2^{66}$ & $2^{255.6}$ & {$[5]$} \\
\cline { 2 - 6 } & 11 & IntA & $2^{120}$ & $2^{150.5}$ & Sec3.2 \\
\cline { 2 - 6 } & 12 & SLA & $2^{66}$ & $2^{249.6}$ & {$[3]$} \\
\cline { 2 - 6 } & 12 & IntA & $2^{120}$ & $2^{214.3}$ & Sec3.2 \\
\hline
\end{tabular}

Note 1. D-Rounds: Distinguisher Rounds; SA: Square Attack; IntA: Integral Attack; HODC: Higher Order Differential Attack; SLA: Square Like Attack.

Note 2. Time complexity is measured in encryption units.

According to Table 1, the integral attacks presented in this paper make significant improvements on both data and time complexities. However, the full-round Camellia provides a sufficient safety margin. Our new method also can be used for any Feistel-SP structure, which raises a natural open problem: How to evaluate the security of Feistel-SP structure against integral attack? This will be our future work.

\section{ACKNOWLEDGMENTS}

We would like to thank Dr. Bozhan Su and Dr. Jian Zou for their helpful comments and suggestions. The research presented in this paper is supported by the National Natural Science Foundation of China (No.60873259 and No. 60903212), and the Foundation Research Funds for the Central Universities (YQNJ1003).
[2] Daemen, J., Knudsen, L., and Rijmen, V.: The block cipher Square. In: Biham, E.(ed.) FSE 1997. LNCS, vol. 1267, pp. 149-165. Springer, Heidelberg (1997)

[3] Duo, L., Li, C., and Feng, K.Q.: Square Like Attack on Camellia. In: Qing, S., Imai, H., and Wang, G.(eds.) ICICS 2007. LNCS, vol. 4861, pp. 269-283. Springer, Heidelberg (2007)

[4] Ferguson, N., Kelsey, J., Lucks, S., Schneier, B., Stay, M., Wagner, D., Whiting, D.: Improved cryptanalysis of Rijndael. In: Schneier, B.(ed.) FSE 2000. LNCS, vol. 1978, pp. 213-230. Springer, Heidelberg (2001)

[5] Hatano, Y., Sekine, H., Kaneko, T.: Higher order differential attack of Camellia (II). In: Nyberg, K., Heys, H.M.(eds.) SAC 2002. LNCS, vol. 2595, pp. 129-164. Springer, Heidelberg (2003)

[6] International Standardization of Organization (ISO), International Standard-ISO/IEC 18033-3, Information technology-Security techniques-Encryption algorithms-Part 3: Block ciphers, July, 2005.

[7] Kanda, M., Matsumoto., T.: Security of Camellia against truncated differential cryptanalysis. In: Matsui, M. (ed.) FSE 2001. LNCS, vol. 2355, pp. 119-137. Springer, Heidelberg (2002)

[8] Knudsen, L. and Wagner, D.: Integral cryptanalysis. In: Daemen, J., Rijmen, V.(eds.) FSE 2002. LNCS, vol. 2365, pp. 112-127. Springer, Heidelberg (2002)

[9] Lee, S., Hong, S., Lee, S., Lim, J., and Yoon, S.: Truncated differential cryptanalysis of Camellia. In: Kim, K.-c.(ed.) ICISC 2001. LNCS, vol. 2288, pp. 32-38. Springer, Heidelberg (2002)

[10] Li, Y., Wu, W., and Zhang, L.: Integral Attacks on Reduced-Round ARIA Block Cipher. In: Kwak, J., et al. (eds.) ISPEC 2010. LNCS, vol. 6047, pp. 19-29, Springer, Heidelberg (2010)

[11] Li, Y., Wu, W., Zhang, L., and Zhang, L.: Improved Integral Attack on Rijndael. Journal of Information Science and Engineering (JISE), to appear.

[12] Reza Z'aba, M., and Raddum, H., Henricksen, M., and Dawson, E.: Bit-Pattern Based Integral Attack. In: Nyberg, K. (ed.), FSE 2008. LNCS, vol. 5086, pp. 363-381. Springer, Heidelberg (2008)

[13] Shirai, T., Kanamaru, S., and Abe, G.: Improved upper bounds of differential and linear characteristic probability for Camellia. In: Daemen, J., Rijmen, V. (eds.) FSE 2002. LNCS, vol. 2365, pp. 147-151. Springer, Heidelberg (2002)

[14] Shirai, T.: Differential, linear, boomerang and rectangle cryptanalysis of reduced-round Camellia. In: Proceedings of 3rd NESSIE workshop (November 2002)

[15] Sugita, M., Kobara, K., and Imai, H.: Security of reduced version of the block cipher Camellia against truncated and impossible differential cryptanalysis. In: Boyd, C. (ed.) ASIACRYPT 2001. LNCS, vol. 2248, pp. 193-207. Springer, Heidelberg (2001)

[16] Wu, W., Feng, D., and Chen, H.: Collision attack and pseudorandomness of reduced round Camellia. In: Handschuh, H., Hasan, M.A.(eds.) SAC 2004. LNCS, vol. 3357, pp. 256-270. Springer, Heidelberg (2004)

[17] Wu, W., Zhang, W. and Feng, D.: Impossible differential cryptanalysis of Reduced-Round ARIA and Camellia. In: Journal of Compute Science and Technology 22(3), pp. 449-456, Springer, Heidelberg (2007)

[18] Wu, W., Zhang, L., and Zhang, W.: Improved impossible differential cryptanalysis of reduced-round Camellia. In: Avanzi, R., Keliher, L., Sica, F. (eds.) SAC 2008. LNCS, vol. 5381, pp. 442-456. Springer, Heidelberg(2009)

[19] Yeom, Y., Park, S., and Kim, I.: On the security of Camellia against the Square attack. In: Daemen, J., Rijmen, V. (eds.) FSE 2002. LNCS, vol. 2365, pp. 89-99. Springer, Heidelberg (2002)

[20] Yeom, Y., Park, S., and Kim, I.: A study of integral type cryptanalysis on Camellia. In Proceedings of the 2003 Symposium on Cryptography and Information Security, pp. 453-456 (2003)

\section{REFERENCES}

[1] Aoki, K., Ichikawa, T., Kanda, M., et al.: Camellia: A 128-Bit block cipher suitable for multiple platforms design and analysis. In: Ito, T., Abadi, M. (eds.) TACS 1997. LNCS, vol. 1281, pp. 39-56. Springer, Heidelberg (1997) 